\title{
Editorial
}

\section{Tropical Protozoan Diseases: Natural Product Drug Discovery and Development}

\author{
Liliana Muschietti, ${ }^{1}$ Roser Vila, ${ }^{2}$ Valdir Cechinel Filho, ${ }^{3}$ and William Setzer ${ }^{4}$ \\ ${ }^{1}$ Department of Pharmacognosy, Faculty of Pharmacy and Biochemistry, \\ University of Buenos Aires, 1113 Buenos Aires, Argentina \\ ${ }^{2}$ Unitat de Farmacologia i Farmacognòsia, Facultat de Farmàcia, Universitat de Barcelona, \\ 08028 Barcelona, Spain \\ ${ }^{3}$ Universidade do Vale do Itajaí, Núcleo De Investigações Químico-Farmacêuticas (NIQFAR), \\ CCS, Brazil \\ ${ }^{4}$ Department of Chemistry, University of Alabama in Huntsville, Huntsville, AL 35899, USA
}

Correspondence should be addressed to Liliana Muschietti; lmusch@ffyb.uba.ar

Received 22 October 2013; Accepted 22 October 2013

Copyright (C) 2013 Liliana Muschietti et al. This is an open access article distributed under the Creative Commons Attribution License, which permits unrestricted use, distribution, and reproduction in any medium, provided the original work is properly cited.

Infections caused by protozoan parasites such as Chagas disease, human African trypanosomiasis, leishmaniasis, or malaria are responsible for considerable morbidity and mortality worldwide with devastating social and economic consequences (Table 1) [1]. Under normal circumstances (efficient epidemiological surveillance programs and sanitary education) the control of these diseases can be carried out effectively. Nevertheless, the implementation of an adequate health care system to palliate the necessities of the affected populations is hindered by the lack of financial and human resources, political instability in these countries, and often questionable government prioritization [2].

Currently, there is lack of effective, safe, and affordable therapies for the treatment of these diseases. The drugs used are far from ideal, and many of them were introduced decades ago; thus the development of new and more effective drugs with fewer side effects represents a crucial dare.

Over the last century, natural products have provided molecules with high structural diversity and "drug-like" properties from a physicochemical point of view. The reason resides in their chemical and steric complexity since they have well-defined three-dimensional structures, improved in terms of efficiency and selectivity for the molecular target $[3,4]$. Natural product research has made a significant contribution to the chemotherapy of parasitic diseases such as quinine and artemisinin whose analogs are currently in use for the treatment of malaria.

This special issue brings significant works, done by leading scientists, and provides an overview on and an insight into recent advances that will contribute to the discovery of natural compounds with high potential against these protozoan parasites.

The readers will find, in nine papers, not only a wide range of topics including the identification of natural compounds with in vitro and in vivo activity against Trypanosoma cruzi, Plasmodium falciparum, or Leishmania spp. but also recent advances in the mechanism of action of bioactive compounds and the design of semisynthetic derivatives as new more effective chemotherapeutic agents with less toxicity. 
TABLE 1: Protozoan infectious diseases.

\begin{tabular}{|c|c|c|c|c|}
\hline Parasites & Disease & Occurrence & Mortality & DALYs \\
\hline Plasmodium spp. & Malaria & 219 million new cases ${ }^{*}$ & 660,000 deaths ${ }^{*}$ & $33,976,000$ \\
\hline Leishmania spp. & Leishmaniasis & $\begin{array}{l}300,000 \text { new cases of VL each year } \\
1 \text { million new cases of CL each year }\end{array}$ & Approx. 40,000 deaths due to VL & $1,974,000^{* *}$ \\
\hline \multirow{2}{*}{ Trypanosoma spp. } & Human African trypanosomiasis & 20,000 actual cases & & $1,673,000^{* *}$ \\
\hline & Chagas disease & Approx. 8 million cases ${ }^{* * *}$ & 12,000 & $430,000^{* *}$ \\
\hline
\end{tabular}

VL: visceral leishmaniasis, CL: cutaneous leishmaniasis, DALY's: Disability Adjusted Life Years.

${ }^{*}$ In 2010, according to the World Malaria Report 2012. ${ }^{* *}$ The Global Burden of Disease Report, WHO, 2004. ${ }^{* * *}$ Second WHO Report on Neglected Tropical Diseases 2013.

\title{
Acknowledgment
}

We are most grateful to the authors for their generous and timely response in spite of their research and academic responsibilities.

\author{
Liliana Muschietti \\ Roser Vila \\ Valdir Cechinel Filho \\ William Setzer
}

\section{References}

[1] DNDi, "Drugs for neglected diseases initiative," http://www .dndi.org/.

[2] V. P. Sülsen, F. M. Frank, S. I. Cazorla et al., "Trypanocidal and ieishmanicidal activities of sesquiterpene lactones from Ambrosia tenuifolia sprengel (Asteraceae)," Antimicrobial Agents and Chemotherapy, vol. 52, no. 7, pp. 2415-2419, 2008.

[3] A. Ganesan, "The impact of natural products upon modern drug discovery," Current Opinion in Chemical Biology, vol. 12, no. 3, pp. 306-317, 2008.

[4] G. M. Cragg and D. J. Newman, "Natural products: a continuing source of novel drug leads," Biochimica et Biophysica Acta, vol. 1830, no. 6, pp. 3670-3695, 2013. 


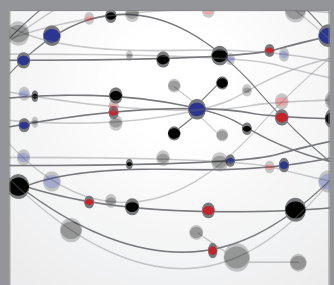

The Scientific World Journal
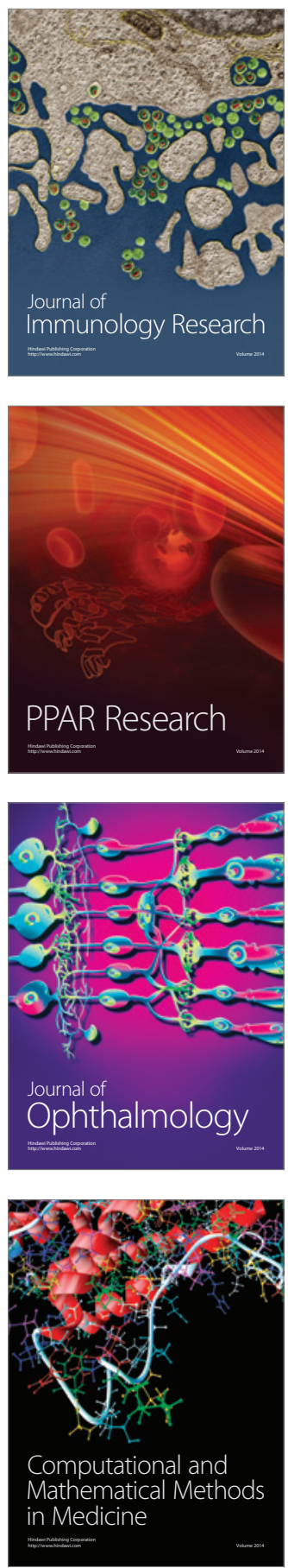

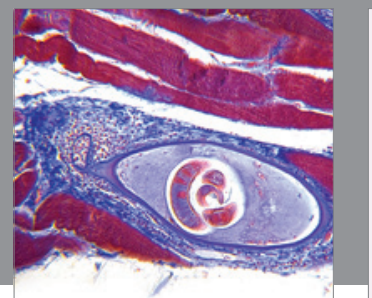

Gastroenterology

Research and Practice
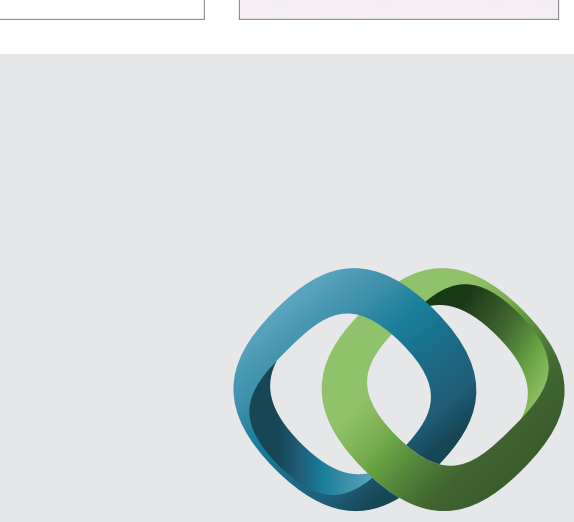

\section{Hindawi}

Submit your manuscripts at

http://www.hindawi.com
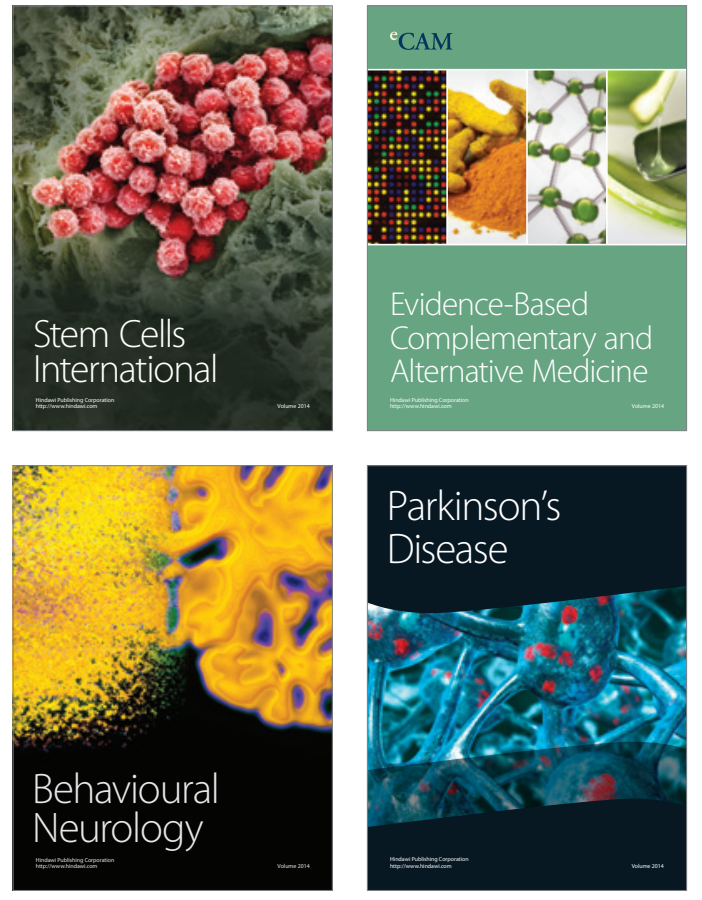
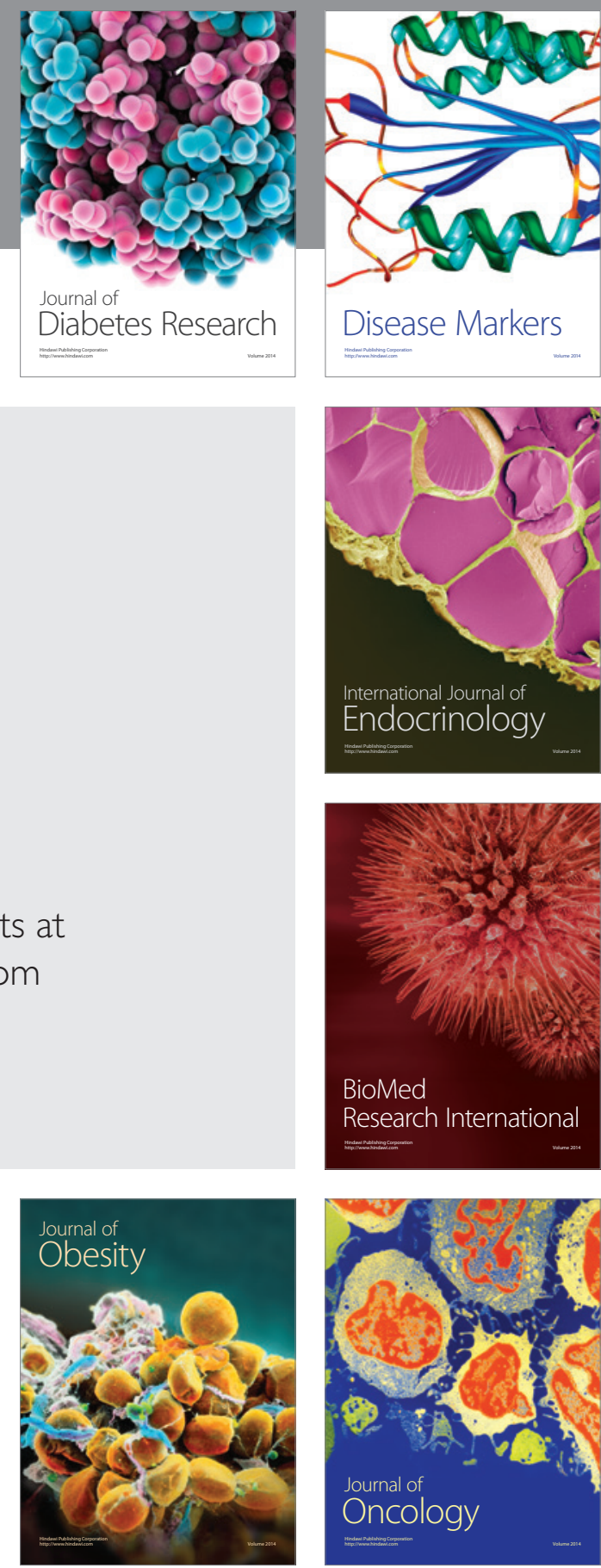

Disease Markers
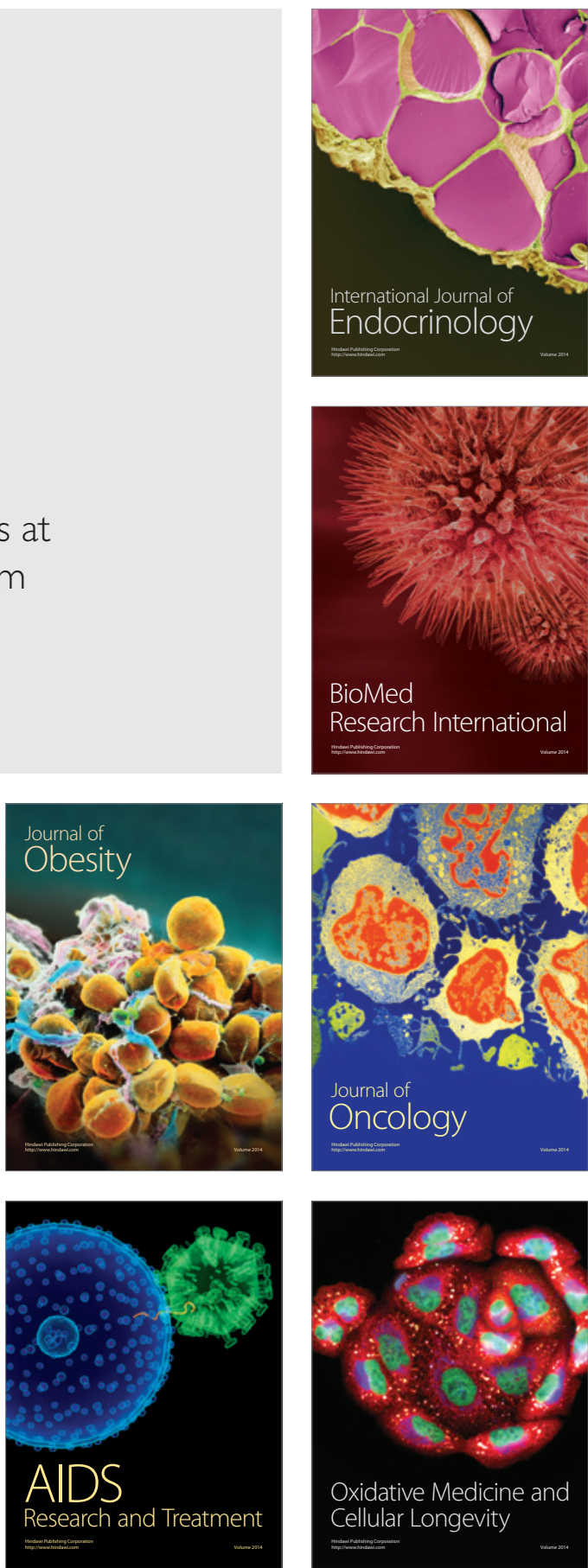